\title{
La valoración de los servicios ecosistémicos desde la cosmovisión indígena totonaca
}

\section{The valuation of ecosystem services from the Totonac indigenous worldview}

Marco Andrés López Santiago ${ }^{*}$

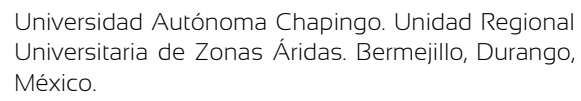

* Autor de correspondencia

marcoandres@chapingo.uruza.edu.mx

\section{RESUMEN}

La cultura totonaca se caracteriza por tener una riqueza en conocimientos sobre el uso y gestión de sus recursos naturales; no obstante, debido a varios factores, se ha visto una disminución en la conservación y transmisión de tales conocimientos. Por tanto, el objetivo principal de este trabajo fue describir los diferentes servicios ecosistémicos de la flora útil del territorio de una población totonaca, con el fin de contribuir a la preservación de los conocimientos tradicionales de la zona en estudio. Se eligió como caso de estudio al municipio de Filomeno Mata, Veracruz, México. Este municipio es característico de la región serrana del totonacapan. Para ello, en el año 2016 se aplicaron entrevistas de tipo semiestructuradas a la población en estudio. Se tuvieron pláticas con informantes clave y un recorrido de campo mediante un transecto dirigido que sirvió para la observación y colecta de las especies. Se encontró que los servicios ecosistémicos más reconocidos por la población se agrupan en cuatro categorías; en orden de importancia los más nombrados se ubicaron en la categoría aprovisionamiento, información, hábitat y regulación, respectivamente. Se concluyó en la necesidad de hacer estudios multidisciplinarios en las comunidades indígenas para el rescate y preservación del conocimiento valioso sobre el uso del capital natural, así como el entendimiento de la asociación indisoluble entre la cultura y los ecosistemas. Las acciones locales para proteger y restaurar ecosistemas benefician tanto a los usuarios directos como a los indirectos a escala nacional o internacional.

PALABRAS CLAVE: capital natural; conocimiento ecológico tradicional; valor sociocultural; Veracruz.

\section{ABSTRACT}

The Totonac culture is characterized by having a richness of knowledge about the use and management of natural resources; nevertheless, due to several factors, there has been a decrease in the conservation and transmission of such knowledge. Hence, the main objective of this work was to describe the different ecosystem services of the flora of a Totonac population, in order to contribute to the preservation of traditional knowledge. The municipality of Filomeno Mata, Veracruz, Mexico was chosen as the case study. This municipality is characteristic of the mountain region of totonacapan. To meet the objective, semi-structured interviews were applied to the target population in 2016. There were talks with key informants and a field trip through a directed transect that served for the observation and collection of the species. It was found that the ecosystem services most recognized by the population are grouped into four categories; in order of importance, the most named were placed in the category of supply, information, habitat and regulation, respectively. It was concluded that there is a need to conduct multidisciplinary studies in indigenous communities for the rescue and preservation of valuable knowledge about the use of natural capital, as well as the understanding of the indissoluble association between culture and ecosystems. Local actions to protect and restore ecosystems, benefit both direct and indirect users at the national or international scale.

KEYWORDS: natural capital; traditional ecological knowledge; socio-cultural value; Veracruz. 


\section{INTRODUCCIÓN}

La naturaleza genera numerosos bienes y servicios para el bienestar humano. Algunos de los beneficios que generan los ecosistemas se obtienen a través de los mercados, mientras que otros son consumidos o disfrutados por los humanos sin la mediación de transacciones mercantiles (Gómez-Baggethun y de Groot, 2007). En este sentido, es importante distinguir entre los procesos y las funciones de los ecosistemas con los servicios ecosistémicos (SE); los primeros describen las relaciones biofísicas que existen independientemente de si los humanos se benefician o no; por el contrario, los servicios ecosistémicos son las características, funciones o procesos ecológicos que contribuyen directa o indirectamente al bienestar humano (Costanza et al., 2017). En términos más simples, los SE son los beneficios que las personas obtienen del funcionamiento de los ecosistemas (Costanza et al., 2017; Millennium Ecosystem Assessment [MEA], 2005).

Esta última definición significa que los beneficios potenciales asociados a las funciones de los ecosistemas se concretizan en beneficios reales una vez que son demandados, usados o disfrutados por las personas; esto es, una vez que las sociedades humanas les asignan valores instrumentales (Gómez-Baggethun y de Groot, 2007). De esta manera, los ecosistemas que proporcionan los servicios se denominan a veces "capital natural", utilizando la definición general de capital como un activo que produce un flujo de servicios a lo largo del tiempo (Costanza et al., 2017).

Por consiguiente, se puede afirmar que los ecosistemas proporcionan una amplia gama de beneficios a la sociedad humana que son de gran valor ecológico, sociocultural y económico (Costanza et al., 1997; de Groot, Wilson y Boumans, 2002; de Groot, 2006; Costanza, 2014).

La dependencia humana de los ecosistemas se aprecia de manera evidente en economías de subsistencia ligadas al medio natural, donde las comunidades humanas toman directamente de los ecosistemas todo lo que necesitan para vivir (Gómez-Baggethun y de Groot, 2007). En particular, los grupos indígenas son poseedores de un gran conocimiento sobre el uso de los recursos naturales (Prieto, 2004), lo que algunos autores han denominado "conocimiento ecológico tradicional" o "conocimiento ecológico local” (Reyes y Martí, 2007). Para esta población, el conocimiento, la clasificación, los usos materiales y las características mágico-religiosas de las plantas no están separados; existe un entrecruzamiento entre los usos materiales de las plantas con sus significados culturales (Bañuelos y Salido, 2007). No obstante, actualmente subsiste una amenaza a la biodiversidad de las áreas rurales, ocasionado principalmente por las altas tasas de deforestación, la ampliación de la frontera agrícola y la contaminación del suelo y agua (Jiménez, Hernández, Espinosa, Mendoza y Torrijos, 2015).

Por otro lado, debido a los vínculos tan estrechos entre la naturaleza con la diversidad cultural en los territorios de poblaciones indígenas o pueblos (Ríos, Alanís y Favela, 2017) sugieren que la pérdida de diversidad cultural constituye un factor de pérdida potencial de diversidad biológica (Jiménez et al., 2015; Reyes y Martí, 2007). Con el fin de analizar las diversas opciones de uso de la tierra, así como los valores ecológicos, económicos y socioculturales, deben combinarse los procedimientos analíticos de valoración con las técnicas de participación de los actores involucrados (de Groot, 2006; Hein, van Koppen, de Groot y van Ierland, 2006). En consecuencia, es importante contar con la información y participación de las comunidades rurales indígenas, no solo para la toma de decisiones del uso de la tierra sino también para la preservación de los conocimientos tradicionales de los servicios ecosistémicos. En este sentido, Bañuelos y Salido (2007) también señalan la pertinencia de llevar a cabo una práctica verdaderamente interdisciplinaria, que no solo registre y describa los conocimientos de los grupos indígenas, sino también intente comprender la lógica que existe detrás de los usos de las plantas. Asimismo, Martínez, Evangelista, Basurto y Mendoza (2002) apuntan que las acciones prioritarias para salvaguardar la riqueza de recursos fitogenéticos deberán ser llevadas a cabo con los campesinos indígenas o mestizos, pues cada grupo o etnia tienen saberes muy 
particulares que pueden definirse como estilos étnicos del conocimiento y manejo de la biodiversidad.

Finalmente, debido a que la valoración de los servicios ecosistémicos se determina analizando el efecto de los ecosistemas y la biodiversidad en el bienestar humano, es necesario comprender las formas en que la sociedad se beneficia de la naturaleza y, por lo tanto, las diversas razones por las que las sociedades valoran los SE. La identificación de los motivos y las motivaciones para proteger los servicios de los ecosistemas ayuda a comprender qué servicios son relevantes para los diferentes actores involucrados (Martín et al., 2012).

\section{OBJETIVOS}

El objetivo principal de este trabajo fue describir e interpretar, desde el enfoque de los actores involucrados, los diferentes servicios ecosistémicos derivados de la flora útil del territorio de una población totonaca. Lo anterior con el fin de contribuir a la preservación de los conocimientos tradicionales de la población en estudio.

\section{MATERIALES Y MÉTODOS}

\section{Ubicación de la zona de estudio}

El estudio se llevó a cabo en el municipio de Filomeno Mata, Veracruz, México. Se sitúa en los paralelos $20^{\circ} 10^{\prime}$ y $20^{\circ} 16^{\prime}$ de latitud norte; los meridianos $97^{\circ} 38^{\prime}$ y $97^{\circ} 45^{\prime}$ de longitud oeste; altitud entre $194 \mathrm{~m}$ y $800 \mathrm{~m}$ snm. Colinda al Norte con los municipios de Coahuitlán, Coyutla y Mecatlán, Veracruz; al Este con el municipio de Mecatlán y el Estado de Puebla; al Sur con el Estado de Puebla; al Oeste con el Estado de Puebla y el municipio de Coahuitlán (Fig. 1). Su clima es semicálido húmedo con lluvias todo el año $(92 \%)$ y cálido húmedo con lluvias todo el año (8\%); presenta un intervalo de temperatura que oscila entre los $20{ }^{\circ} \mathrm{C}-26{ }^{\circ} \mathrm{C}$. La precipitación se encuentra entre los $2900 \mathrm{~mm}$ y los $3100 \mathrm{~mm}$. Cuenta con una superficie de $43.2 \mathrm{~km}^{2}$, lo que representa $0.1 \%$ de la superficie del Estado de Veracruz. Para 2010, presentó una densidad poblacional de 424.8 habitantes por kilómetro cuadrado (Secretaría de Finanzas y Planeación del Estado de Veracruz [Sefiplan], 2018).
En cuanto a la fisiografía, forma parte de la provincia de la Sierra Madre Oriental, la Subprovincia Carso Huasteco (discontinuidad fisiográfica) y el sistema de topoformas sierra alta escarpada. Los ecosistemas que coexisten en el municipio son del tipo bosque subtropical perennifolio (Instituto Nacional de Estadística y Geografía [Inegi], 2009).

El municipio cuenta con un total de 276 parcelas que suman un total aproximado de 1300 ha, todas bajo el régimen de pequeña propiedad (Inegi, 2009). Para 2016, contó con una superficie total sembrada de 1289.5 ha, de las cuales 715 ha fueron maíz grano, 553 ha correspondieron a café cereza y 20 ha a pimienta (Sefiplan, 2018).

De acuerdo con datos de la Sefiplan (2018), para 2015, el municipio tenía una población total de 18367 habitantes, de los cuales $42.54 \%$ era poblacion infantil (0 - 14 años), $53.15 \%$ era joven y adulta (15 años - 64 años) y $4.25 \%$ correspondía a la tercera edad (65 años y más). Del total de la población de 3 años y más, $97.26 \%$ habla la principal lengua indígena que es la totonaca.

Es un municipio característico de la región serrana del totonacapan. La cultura totonaca y en particular la zona de estudio se caracteriza por tener una riqueza en conocimientos empíricos sobre el uso y gestión de sus recursos naturales; no obstante, debido al incremento poblacional, el proceso de urbanización desordenada y la migración (nacional e internacional), ha visto una disminución en la conservación y transmisión de tales conocimientos.

\section{Selección y tamaño de muestra}

La población objetivo fueron personas mayores de 55 años que ya poseen los conocimientos de uso y localización de las especies. Se determinó concentrarse en este estrato de la población debido que varios autores señalan que es la población de mayor edad la que conoce el uso y la identificación de plantas útiles en una comunidad (Bautista, Sol, Velázquez y Llanderal, 2016; Gómez, Sol, García y Pérez, 2016). 


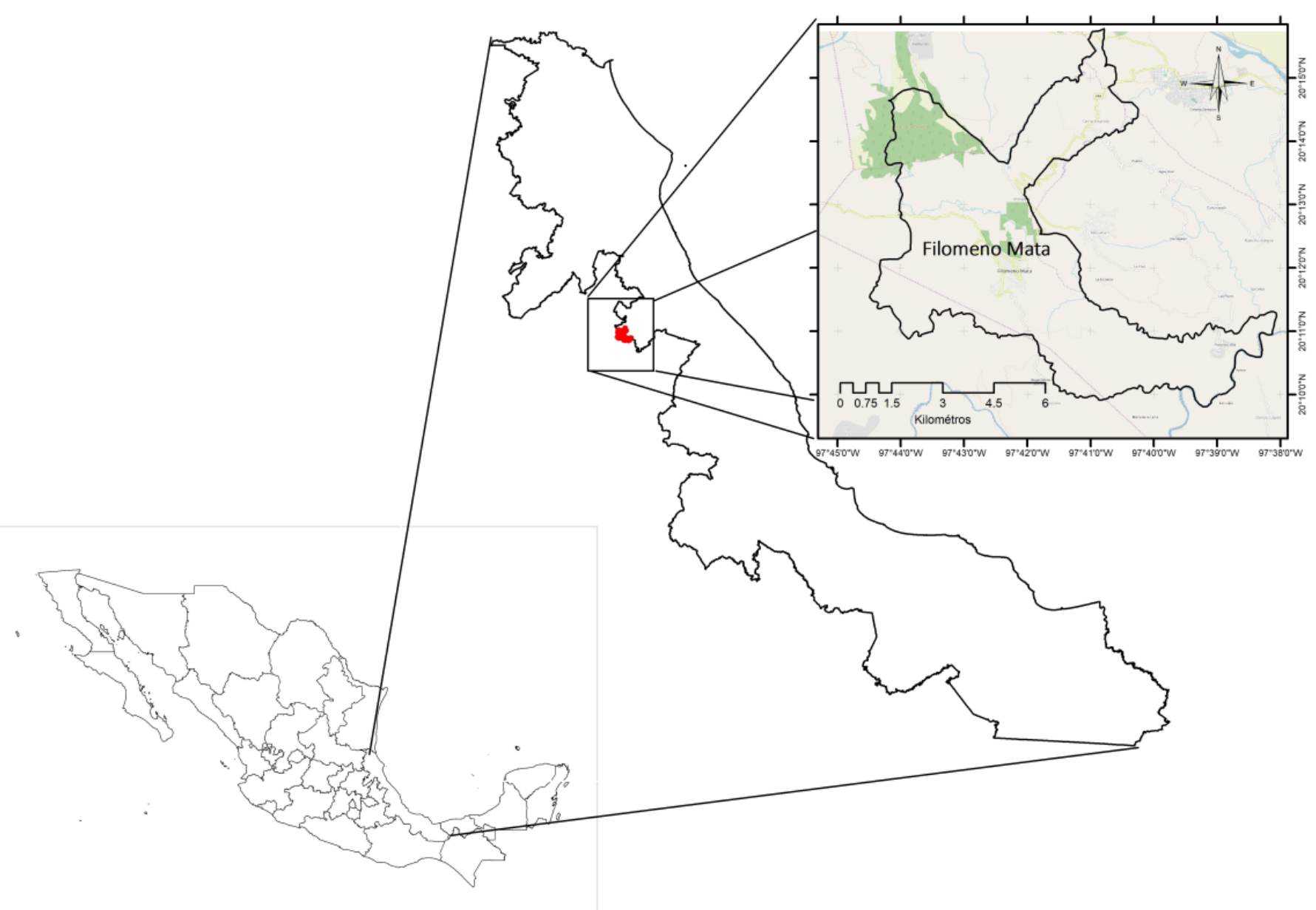

FIGURA 1. Ubicación de la zona de estudio.

Las entrevistas fueron de tipo semiestructuradas y se aplicaron mediante un muestreo aleatorio simple. Para calcular el tamaño de la muestra se utilizó la siguiente fórmula para poblaciones finitas (Anderson, Sweeney y Williams, 2008):

$$
n=\frac{N * Z_{\alpha}^{2} * p * q}{d^{2} *(N-1)+Z_{\alpha}^{2} * p * q}
$$

Donde:

$\mathrm{n}=$ tamaño de la muestra

$\mathrm{N}=$ población total del universo de estudio: 980 personas

$\mathrm{p}=0.05$

$\mathrm{q}=1-\mathrm{p}$

$\mathrm{E}=$ error o precisión de estimación permitido $(6 \%)$

$\mathrm{Z}=1.645$
$\mathrm{Al}$ sustituir los datos resultó un total de 34.48 encuestas; sin embargo, cuatro de las encuestas tuvieron inconsistencias, por lo que se trabajaron con 30 cuestionarios. A los informantes se les cuestionó sobre el conocimiento de las plantas de la comunidad, el uso que le dan, el manejo, nombres en español y nombres en totonaco de las plantas. El cuestionario se estructuró en cinco apartados con un total de 15 preguntas. Paralelamente, se realizó un recorrido de campo mediante un transecto dirigido para la colecta de las plantas reportadas. Con el apoyo de tesistas participantes en el proyecto, se identificaron los ejemplares recolectados mediante el uso de claves taxonómicas y se compararon las muestras colectadas con las que se encuentran en los herbarios de la Universidad Autónoma Chapingo. Se 
verificaron las vigencias de la nomenclatura botánica mediante la página de internet www.trópicos.com.mx.

Ahora bien, un paso importante para el análisis de los servicios ecosistémicos implica la traducción de la complejidad ecológica (estructuras y procesos) en un número más limitado de funciones del ecosistema (de Groot, 2006). Al respecto, para la clasificación de dichas funciones y servicios ecosistémicos existen muchos criterios (Costanza, 1997; Daily, 2000; de Groot et al., 2002; MEA, 2005); sin embargo, uno de los primeros marcos estandarizados para el análisis de funciones y servicios se encuentra en de Groot et al. (2002) que provee una lista de 23 funciones (agrupadas en cuatro grandes grupos), además, enumera las estructuras ecológicas y los procesos subyacentes a estas funciones y finalmente proporciona una lista más detallada con ejemplos de bienes y servicios específicos derivados de dichas funciones. Por esta razón, en este trabajo se utilizó el criterio de clasificación de estos últimos autores (Apéndice 1).

Para cada una de las funciones y servicios de los ecosistemas se analizaron en particular las especies que las generan, asimismo se describieron los beneficios a la población desde la lógica inherente a la cosmovisión indígena totonaca. Como una innovación en este trabajo, para las especies se escribe su nombre científico, nombre común en español y en lengua indígena totonaca.

\section{Resultados}

\section{Función regulación}

En la descripción de la flora que utilizan los totonacos de la zona de estudio como función de regulación, se les preguntó sobre las especies y la forma en que ellos creen que beneficia tanto a sus cultivos como al suelo. En este sentido, los pobladores explicaron que se utiliza normalmente el sistema roza-tumba-quema para la primera ocasión en que se va a sembrar en una parcela. En el siguiente ciclo de siembra para la misma parcela solo se lleva a cabo la roza, se dejan crecer algunas especies forestales como el jonote (Heliocarpus appendiculatus Turcz), sangre de grado (Croton draco Schltdl. \& Cham.), chalahuite (Inga jinicuil Schltdl) y cuerillo (Trema micrantha (L.) Blume). A estos arbustos se les realizan podas de mantenimiento por los siguientes tres años. Los pobladores afirmaron que las hojas y ramas que resultan de la poda se pudren en el suelo, con ello se favorece el mantenimiento de los nutrientes y la formación de los suelos. Pasados los tres años de mantenimiento, las especies descritas se tumban para utilizarse como combustible; no obstante, se dejan algunos troncos de jonote (Heliocarpus appendiculatus) para que continúe el proceso de formación de suelos (para los nombres en totonaco véase la tabla 1).

Para el caso del Inga jinicuil Schltdl, Vargas y Pire (2017) mencionan que la planta mejora las condiciones del suelo al aportar nutrientes y materia orgánica de las hojas y ramas, y por el estimulo que producen sobre la actividad microbiana del suelo. En recuperación de tierras degradadas, es una especie ideal por su aporte de hojarasca y manejo bajo podas. La hojarasca presenta contenidos moderados de nitrógeno y altos de lignina y polifenoles, por lo cual presenta una descomposición lenta y no ofrece ciclos rápidos como abono verde, pero puede proporcionar una acumulación a largo plazo de nitrógeno orgánico y control efectivo de malezas.

TABLA 1. Ejemplos especies como funciones de regulación

\begin{tabular}{ll}
\hline Función & Especies que generan los bienes y servicios \\
\hline Retención de suelo & Jonote o xunak (Heliocarpus appendiculatus Turcz.); sangre de grado o puklhn (Croton \\
Formación de suelos & draco Schltdl. \& Cham.); chalahuite o kalam (Inga jinicuil Schltdl.); cuerillo o chakgat \\
Regulación de nutrientes & (Trema micrantha (L.) Blume) \\
\hline
\end{tabular}


En la región de estudio, los terrenos de cafetales, así como los agostaderos son cercados por plantas de cocoíte (Gliricidia sepium (Jacq.) Kunth ex Walp), chaka (Bursera simaruba (L.) Sarg.) y pichoco (Erythrina americana Mill).

\section{Función hábitat}

Con respecto a las especies que cumplen con la función hábitat, se mencionó que anteriormente cuando abundaban los tucanes y loros, ellos preferían posarse sobre el chalahuite (Inga jinicuil Schltdl) y otros árboles frutales. Les gustaba posarse sobre estos árboles ya que de ahí se alimentaban. El chalahuite (Inga jinicuil Schltdl) todavía funciona como refugio del pájaro carpintero, además es utilizado para dar sombra a las plantas de café (Coffea arabica L). Por su parte, el pichoco (Erythrina americana Mill) cumple la función de hospedero para algunas orquídeas.

Hoy en día existen especies valoradas por los totonacos debido a que cumplen con la función criadero de flora y fauna comestible. Por ejemplo, durante el proceso de degradación, las especies que funcionan como criadero de hongos comestibles son la chaka (Bursera simaruba (L.) Sarg.), jonote (Heliocarpus appendiculatus Turcz) y hormiguilla (Cecropia obtusifolia Bertol.). Asimismo, la hojarasca que forma el encino (Quercus elliptica Née) en el suelo también favorece la formación de hongos (para los respectivos nombres en totonaco véase la tabla 2).

Además de producir hongos comestibles en su proceso de degradación, el jonote (Heliocarpus appendiculatus Turcz) en pie o vivo, cumple con la función de criadero de la larva conocida en la región como cuetla (Arsenura armida). Los cuetlas se alimentan de las hojas del jonote. Estas larvas son recolectadas y consumidas por la población en temporada de lluvias; aunque actualmente pocas familias continúan con el consumo de estas larvas.

\section{Función de producción}

En relación con la función de producción, se encontró una gran variedad de ejemplos de bienes y servicios. Para su análisis se agruparon en: comestibles, medicinales, combustibles, maderables y construcción, forrajeras, decorativos, embalaje y artesanías.

Se encontró que 64 de las 110 especies descritas por las personas son comestibles. De acuerdo con la frecuencia mencionada, las especies silvestres más consumidas son el tomate silvestre (Lycopersicon lycopersicum L. H. Karst.), hierba mora (Solanum nigrum L.), calabaza (Cucurbita argyrosperma $\mathrm{K}$. Koch) y el chile de árbol (Capsicum annum L.).

La mayoría de las especies son silvestres y se obtienen por recolección dentro de los cultivos de maíz o en el monte, tal es el caso de la hierba mora (Solanum nigrum L.), tomatillo (Physalis gracilis Miers), jaboncillo (Phytolacca rivinoides Kunth \& C.D. Bouché), quelite blanco (Amaranthus cruentus L.); quelite quintonil (Amaranthus bypochondriacus L.), malvarón (Xanthosoma robustum Schott), verdolaga (Portulaca oleracea L.) y el bigote de acamayo (Smilax laceolata L.) (para los respectivos nombres en totonaco véase la tabla 3).

Las especies descritas son consideradas como "verduras silvestres" por la población. Las personas entrevistadas afirmaron que estas plantas se siguen consumiendo porque tienen la confianza de que son de origen natural, además las encuentran en sus terrenos de cultivo.

TABLA 2. Ejemplos especies como funciones hábitat

\begin{tabular}{ll}
\hline Función & Especies que generan los bienes y servicios \\
\hline Función refugio & $\begin{array}{l}\text { Chalahuite o kalam (Inga jinicuil Schltdl.); pichoco o Ihalhni (Erythrina americana Mill.); chaka } \\
\text { o tasunn (Bursera simaruba (L.) Sarg.); jonote o xunak (Heliocarpus appendiculatus Turcz.); } \\
\text { hormiguilla o akgow (Cecropia obtusifolia Bertol.); encino o kúkat (Quercus elliptica Née). }\end{array}$ \\
\hline
\end{tabular}

Fuente: Elaboración propia con base en las encuestas aplicadas 
TABLA 3. Ejemplos de especies como funciones de producción.

\begin{tabular}{|c|c|}
\hline Función & Especies que generan los bienes y servicios \\
\hline
\end{tabular}

Fuente: Elaboración propia con base en las encuestas aplicadas

Otro aspecto que destaca en la función de producción son los frutos silvestres, mismos que también son de recolección. En esta categoría se tiene al capulín (Parathesis psychotrioides Lundell), chalahuite (Inga spuria Humb. \& Bonpl. Ex Willd), anay (Beilschmiedia anay (S.F. Blake) Kosterm.), chirimoya (Annona cherimola Mill.), ciruelo (Spondias purpurea L.), jobo (Spondias mombin L.), niguas (Miconia astroplocama Donn. Sm.), papullo (Gonolobus niger (Cav.) R. Br. Ex Schult.), pomarrosa (Syzygium jambos (L.) Alston), pahua (Persea schiedeana Nees), tepetomate (Pseudolmedia oxyphyllaria Donn. Sm.), zapote cabello (Mammea americana 1.), zapote mante (Pouteria campechiana (Kunth) Baehni), zapote negro (Diospyros digyna Jacq.) (para los respectivos nombres en totonaco véase la tabla 3). En su mayoría, estos frutos exóticos se suelen encontrar entre los cultivos de café (Coffea arabica L.), ya que además de producir frutos comestibles también funcionan como sombra para los cafetales.

Por otro lado, el comercio de estas especies se lleva a cabo de casa en casa, los tianguis, puestos semifijos de la calle principal y mercado del municipio. Las personas de mayor edad son las que consumen principalmente estas frutas y verduras silvestres. Asimismo, estas mismas personas transmiten sus conocimientos sobre el consumo y forma de preparación de los platillos a las nuevas generaciones.

En otro orden de ideas, se encontraron 23 especies medicinales. Algunas son consumidas como infusión 
caliente, tal es el caso de la raíz de hierba santa (Piper auritum Kunth) y las hojas de naranja (Citrus sinensis (L.) osbeck) que son un remedio para el dolor del vientre. Las hojas de cempaxúchitl (Tagetes erecta L.) se muelen con un poco de agua fría, se cuela y el líquido sirve como remedio para el dolor de estómago, mareos, vómitos y parásitos estomacales.

Un remedio tradicional muy común en los hogares es la mezcla de la hierba del golpe (Oenothera rosea L'Hér. Ex
Aiton), maltanzin (Satureja brownei (swartz) Briq.) y alcohol de caña (Saccharum officinarum L.). Esta mezcla se deja reposar por días hasta que el alcohol tome un tomo verdoso-negruzco, se utiliza untando el líquido en el cuerpo del paciente o bien dándole un trago, esta preparación sirve como remedio para las personas que padecen de "mal de aire" o bien dicho que sufren de mareos repentinos y ataques nerviosos (para los respectivos nombres en totonaco véase la tabla 4).

TABLA 4. Ejemplos de especies como funciones de producción.

\begin{tabular}{ll}
\hline Función & Especies que generan los bienes y servicios \\
\hline & Caña o chánkat (Saccharum officinarum L.); brumo o xtankgoyut kgantsális (Smilax \\
& aristolochiifolia Mill); caña de puerco o ix chankat paxni (Costus spicatus (Jacq.) Sw.); \\
& cempaxúchitl o kgalhpuxam (Tagetes erecta L.); chaka o tasunn (Bursera simaruba (L.) Sarg); \\
& chocomitillo o talunkshnukiw (Hamelia patens Jacq.); epazote o Ihkgna (Dysphania ambrosioides \\
& (L.) Mosyakin \& Clemants); guayabo o asiwit (Psidium guajava L.); hierba del golpe o \\
& paxtocnupalhma (Oenothera rosea L'Hér. Ex Aiton); hierba dulce (Lippia dulcis Trevir); hierba \\
& santa o xinán (Piper auritum Kunth); hoja de lantei o xnokgtay (Plantago major L.); hoja morada \\
& con verde o tatlekgeplhma (Tradescantia zebrina Heynh. Ex Bosse); hormiguilla o akgow \\
medicinales & (Cecropia obtusifolia Bertol.); xinan grande o kgonsap (Piper peltatum L.); jonote o xunak \\
& (Heliocarpus appendiculatus Turcz.); mala mujer o kgan (Cnidoscolus multilobus (Pax) I. M. \\
& Johnston); maltanzin o pekuatawan (Satureja brownei (Sw.) Briq.); naranja o laxix (Citrus × \\
& sinensis (L.) Osbeck); sangre de grado o puklhn (Croton draco Schltdl. \& Cham.); tepejilote o \\
& litámpa (Chamaedorea oblongata Mart.); tepozán (Buddleja cordata Kunth); cedro o puksnánkiw \\
& (Cedrela odorata L.); pimienta o ukúm (Pimenta dioica (L.) Merr.).
\end{tabular}

Combustibles

Café o kapéj (Coffea arabica L.); chaka o tasunn (Bursera simaruba (L.) Sarg.); chalahuite o kalam (Inga jinicuil Schltdl.); encino o kúkat (Quercus elliptica Née); guácima o akgaxti (Guazuma ulmifolia Lam.); guayabo o asiwit (Psidium guajava L.); hormiguilla o akgow (Cecropia obtusifolia Bertol.); jonote o xunak (Heliocarpus appendiculatus Turcz.); cuerillo o chakgat (Trema micrantha (L.) Blume); naranja o laxix (Citrus × sinensis (L.) Osbeck); nogma o tsapal (Vernonia patens Kunth); pahua o Ihpaw (Persea schiedeana Nees); palo de agua o káp (Dendropanax arboreus (L.) Decne. \& Planch.); palo de agua o tampokg (Pachira aquatica Aubl.); sangre de grado o puklhn (Croton draco Schltdl. \& Cham.); sauco o tkgxkiw (Sambucus mexicana C. Presl ex DC); timbrillo Materia prima blanco o tsutsún (Calliandra houstoniana (Mill.) Standl.).

Maderable y construcción

Caoba o aksan (Swietenia humilis Zucc.); cedro o puksnánkiw (Cedrela odorata L.); jonote o xunak (Heliocarpus appendiculatus Turcz.); mango o mángu (Mangifera indica L.); cuerillo o chakgat (Trema micrantha (L.) Blume); encino o kúkat (Quercus elliptica Née); tarro o matluk (Guadua angustifolia Kunt).

Forrajeras

Guácima o akgaxti (Guazuma ulmifolia Lam.); jonote o xunak (Heliocarpus appendiculatus Turcz.); mazote o xtuy (Bidens pilosa L); ramón o tankuxalh (Brosimum alicastrum Sw.). 
Del mismo modo, se usa el exudado de sangre de grado (Croton draco Schltdl. \& Cham) y jonote (Heliocarpus appendiculatus Turcz) para cerrar heridas, mientras que las hojas de chocomitillo (Hamelia patens Jacq.) funcionan como sellador para evitar infecciones en heridas abiertas. También la caña de puerco (Costus spicatus (Jacq.) Sw.) es utilizada como remedio para algunos problemas urinarios (para los respectivos nombres en totonaco véase la tabla 4).

En los hogares del lugar de estudio aún se utiliza la leña para cocinar los alimentos y se utilizan diversas especies como combustible. De acuerdo con las entrevistas, cualquier árbol puede ser combustible siempre y cuando esté seco; no obstante, la gente prefiere los árboles o arbustos de rápido crecimiento o acahuales, como son conocidos localmente. Las especies comúnmente usadas para este fin son: sangre de grado (Croton draco Schltdl. \& Cham.), chalahuite (Inga jinicuil Schltdl.), cuerillo (Trema micrantha (L.) Blume), nogma (Vernonia patens Kunth), jonote (Heliocarpus appendiculatus Turcz) y café (Coffea arabica $\mathrm{L}$.).

En relación con las especies maderables y para construcción, destaca la madera de caoba (Swietenia bumilis Zucc.) y cedro (Cedrela odorata L.) como maderas preciosas comercializables. Algunas familias de bajos ingresos siguen construyendo las paredes de sus hogares con madera de jonote (Heliocarpus appendiculatus Turcz.), cuerillo (Trema micrantha (L.) Blume) o tarro (Guadua angustifolia Kunt). El encino (Quercus elliptica Née) se usa para viguetas o postes debido a la dureza de esta madera.

La población totonaca se caracteriza por tener ganado de traspatio como cerdos, gallinas, guajolotes y borregos. Estos animales se alimentan con los desperdicios de la cocina y complementan la dieta con mazote (Bidens pilosa L.) y hojas de jonote (Heliocarpus appendiculatus Turcz.). Además de la ganadería de traspatio, los pobladores usan caballos, mulas y burros para transportar su producción. Estos animales para transporte se alimentan con pasto nativo, mazote (Bidens pilosa L.) y hojas de mazorca de maíz (Zea mays L.).
En cuanto a los recursos genéticos, la población totonaca conserva diferentes tipos de maíz y frijol criollos. Ellos afirman que el maíz producido en su comunidad tiene mejor sabor, color y olor con respecto al maíz comercial.

Respecto a las especies que sirven para decoración, es destacable el uso que se le da a la flora durante la celebración del día de muertos (se celebra del 31 de octubre al 2 de noviembre). Un altar totonaco tradicional se compone de un adorno en forma de "u invertida" decorado con cempaxúchitl (Tagetes erecta L.) y tepejilote (Chamaedorea oblongata Mart). También se usa la combinación de tepejilote (Chamaedorea oblongata Mart) con alguna de las flores de: pasmaxanath (Gomphrena globosa L.), papatla (Heliconia latispatha Benth), labio de mujer (Psychotria elata (Sw.) Hammel) o mano de león (Celosia argentea L.) (para los respectivos nombres en totonaco véase la tabla 5).

Como complemento en el altar para las fechas del 31 de octubre al 2 de noviembre se usan diferentes frutales como mandarina (citrus reticulata Blanco), naranja (Citrus sinensis (L.) Osbeck), camote (Ipomoea batatas (L.) Lam.), calabaza (Cucurbita argyrosperma K. Koch), plátano (Musa acuminata Colla) y caña (Saccharum officinarum L.). Asimismo, en la mesa del altar se ponen los diferentes guisos hechos con las "verduras silvestres" (para los respectivos nombres en totonaco véase la tabla 5).

Después del trabajo en los huertos o milpas, los productores del lugar de estudio tienen la costumbre de recolectar frutos de temporada como el jobo (Spondias mombin L.), capulín (Parathesis psychotrioides Lundell) o tepetomate (Pseudolmedia glabrata (Liebm.) C.C. Berg). Estas frutas silvestres son envueltas en hojas de papatla (Heliconia hirsuta L.f. y Heliconia irrasa Lane ex R.R.Sm.), para ser transportadas hasta los hogares. Del mismo modo, estas especies de plantas son usadas como embalaje para vender los productos recolectados.

También dentro de la función de producción se ubican las especies utilizadas para artesanías. En este sentido, las trozas de caoba (Swietenia humilis Zucc.) y cedro (Cedrela odorata L.) sirven para hacer artesanías como bateas, cucharas, máscaras y juguetes para los niños. En la cocina 
se utilizan los frutos secos de jícaro (Lagenaria siceraria (Molina) Standl.) para servir café. En algunos hogares de escasos recursos económicos, todavía se utiliza el bagazo de la caña como cama. Estos productos artesanales solo se comercializan de manera local y el precio lo define el artesano dependiendo del tiempo que se tarde en elaborarlos.

\section{Función de información}

Finalmente, con relación a la función de información la comunidad de estudio tiene una estrecha relación entre la flora y las festividades, rituales o ceremonias religiosas.

Las especies que se enlistaron en la categoría decorativos también se utilizan para la función espiritual como adorno en las casas en días festivos-religiosos, bodas, bautizos y demás festividades que se celebran en los hogares de los habitantes. Los pobladores son creyentes de los ritos antes de cada celebración y las plantas representan una ofrenda muy importante.
En este mismo sentido, las hojas de hierba santa (Piper auritum Kunth) son utilizadas para bañar a los recién nacidos, así como a las mujeres en cuarentena. El baño con hierba santa se realiza en un temazcal. También en esta categoría mágico-religiosa se ubica a la escobilla (Sida rhombifolia L.), misma que es utilizada para barrer en la iglesia cada viernes santo. Las hojas de palma de coyol (Scheelea liebmannii Becc.) se usan para hacer diferentes figuras como adorno para la iglesia el domingo de Ramos.

La gente también reconoce que sin el café (Coffea arabica L.) su vida no sería la misma. Por una parte, el cultivo fue durante mucho tiempo la única fuente de ingresos; por otro lado es una bebida esencial para las comidas del hogar, ya que se toma café a cualquier hora del día. De acuerdo con los testimonios, una comida popular se compone de una taza de café, tortilla de maíz (Zea mays L.) hecha a mano, un plato de frijoles (Phaseolus vulgaris L.) y una salsa preparada en molcajete con jitomate silvestre (Lycopersicon ycopersicum (L.) H. Karst.) y chiltepin (Capsicum frutescens L.) (para los respectivos nombres en totonaco véase la tabla 6).

TABLA 5. Ejemplos de especies como funciones de producción

\begin{tabular}{|c|c|}
\hline Función & Especies que generan los bienes y servicios \\
\hline Recurso genético & Maíz o kúxi (Zea mays L.); frijol o stapu (Phaseolus vulgaris L.). \\
\hline \multirow{7}{*}{$\begin{array}{l}\text { Recursos } \\
\text { ornamentales }\end{array}$} & Decorativos \\
\hline & $\begin{array}{l}\text { Amor de un rato (Portulaca grandiflora Hook.); blanca mariposa o mariposa xanat } \\
\text { (Hedychium coronarium J. Koenig); buganvilia o Ihpupukg xanat (Bougainvillea glabra } \\
\text { Choisy); flor de corazón o kuwi xanat (Talauma mexicana (DC.) G. Don); flor de papatla o } \\
\text { xuékgtay (Heliconia latispatha Benth); labio de mujer o tampikiw (Psychotria elata (Sw.) } \\
\text { Hammel); mano de león o makgtánkganpins (Celosia argentea L.); muérdago o } \\
\text { makgtahual (Phoradendron nervosum Oliv.); pasmaxanath (Gomphrena globosa L.). }\end{array}$ \\
\hline & Embalaje \\
\hline & Papatla o sekgnápalhm (Heliconia hirsuta L. F.); papatla o sekgnápalhm (Heliconia irrasa \\
\hline & Lane ex R.R. Sm.); maíz o kúxi (Zea mays L.); chikitchi (Heliconia latispatha Benth.). \\
\hline & Artesanías \\
\hline & $\begin{array}{l}\text { Jícaro o pumaksat (Lagenaria siceraria (Molina) Standl.); café o kapéj (Coffea arabica L.); } \\
\text { caoba o aksan (Swietenia humilis Zucc.); cedro o puksnánkiw (Cedrela odorata L.); } \\
\text { mandarina o kapúnesus (Citrus reticulata Blanco); naranja o laxix (Citrus x sinensis (L.) } \\
\text { Osbeck); caña o chánkat (Saccharum officinarum L.). }\end{array}$ \\
\hline
\end{tabular}

Fuente: Elaboración propia con base en las encuestas aplicadas 
TABLA 6. Ejemplos de especies como funciones de información

\begin{tabular}{ll}
\hline Función & Especies que generan los bienes y servicios \\
\hline & Maíz o kúxi (Zea mays L.); café o kapéj (Coffea arabica L.); lekgenun linekgen (Picramnia \\
& teapensis Tul.); hierba santa o xinán (Piper auritum Kunth); palma de coyol o mokgot (Scheelea \\
& liebmannii Becc.); escobilla o akgawana lipalhn (Sida rhombifolia L.); tepejilote o litámpa \\
& (Chamaedorea oblongata Mart.); cempaxúchitl o kgalhpuxam (Tagetes erecta L.); pasmaxanath \\
& (Gomphrena globosa l.); flor de papatla o xuékgtay (Heliconia latispatha Benth.); mano de león o \\
& makgtánkganpins (Celosia argentea L.); labio de mujer o tampikiw (Psychotria elata (Sw.) \\
Información & Hammel); blanca mariposa o mariposa xanat (Hedychium coronarium J.Koenig); camote o mánta \\
histórica y & (Ipomoea batatas (L.) Lam.); caña o chánkat (Saccharum officinarum L.); naranja o laxix (Citrus x \\
espiritual & sinensis (L.) Osbeck); plátano o sekgna (Musa acuminata Colla); mandarina o kapúnesus (Citrus \\
& reticulata Blanco); calabaza o nipx (Cucurbita argyrosperma K. Koch); yuca o kgoxkgewe \\
& (Manihot esculenta Crantz); malvarón o lokg (Xanthosoma robustum Schotr); quelite blanco o \\
& tsaw juki (Amaranthus cruentus L.); quelite quintonil o tsaw (Amaranthus hypochondriacus L.); \\
& hierba mora o mustúlut (Solanum nigrum L.); bigote de acamayo o kgansalis (Smilax laceolata L.); \\
& papatla o sekgnápalhm (Heliconia hirsuta L. F.).
\end{tabular}

Fuente: Elaboración propia con base en las encuestas aplicadas

\section{DISCUSIÓN}

De los tres enfoques de valoración de los SE (ecológico, sociocultural y económico) conceptualizado por varios autores (de Groot et al., 2002; de Groot, 2006; GómezBaggethun y de Groot, 2007; Martín et al., 2012, entre otros), en este trabajo se dio prioridad a la valoración sociocultural. Entendiéndose el valor sociocultural a los aspectos como la educación, la diversidad y la identidad cultural, la libertad y los valores espirituales como factores moldeadores de las preferencias humanas (de Groot et al., 2002; de Groot, 2006; Gómez-Baggethun y de Groot, 2007).

De acuerdo con el análisis del caso de estudio, la población totonaca tiene un conocimiento vasto e importante sobre los SE que se derivan de las funciones ecosistémicas de la flora, mismo que puede contribuir al uso sustentable del capital natural. Por ejemplo, el uso del sistema roza-tumba-quema más que perjudicial para el medio ambiente se percibe en la localidad como apoyo para la generación de la función ecosistémica de regulación. Al respecto, Reyes y Martí (2007) afirman que numerosos estudios de este sistema agrícola han destacado el papel del conocimiento agronómico tradicional en la preservación de multitud de variedades de plantas agrícolas y razas animales.
También, Martínez et al. (2002) sugieren que a veces puede bastar la conservación de los sistemas agrícolas tradicionales y el cultivo de razas criollas para mantener la diversidad in situ. Entonces, es posible afirmar que las formas locales de manejo de los recursos naturales contribuyen a la generación y conservación de la diversidad biológica mediante la manipulación de plantas, animales, hábitats y ecosistemas (Reyes y Martí, 2007).

A pesar de la riqueza del conocimiento que posee la cultura totonaca, la misma población reconoce que los conocimientos sobre el valor y uso del capital natural han ido en decadencia. Se mencionó que unos de los factores en detrimento del conocimiento tradicional podrían ser los cambiantes gustos y preferencias de la población joven. En este sentido, Hein et al. (2006) mencionan que los actores involucrados pueden tener perspectivas muy diferentes sobre los valores de los servicios de los ecosistemas, basados entre otros, en su dependencia de servicios específicos para proporcionar ingresos o mantener su entorno de vida. Asimismo, tanto Martín et al. (2012) como Briceño, Iñiguez y Ravera (2016) sostienen que los SE son valorados de manera diferente por diferentes actores, según el contexto socioeconómico y cultural, así como con base 
en sus preferencias, necesidades, valores, normas y aspiraciones.

Según los informantes, los padres y abuelos fueron los que les cedieron los conocimientos que poseen acerca del uso de la vegetación, tratan de hacer lo mismo con sus hijos y nietos; sin embargo, consideran que es necesaria una difusión más extensa de los usos y costumbres para que las nuevas generaciones revitalicen la importancia e interés que merecen; al respecto Huasasquiche y Kómetter (2017) también afirman que los saberes y conocimientos comunales están desapareciendo debido a la deficiente transmisión de información entre generaciones.

Algunos de los autores relevantes a escala internacional que han utilizado la metodología o enfoque similar abordado en este trabajo se encuentran Martín et al. (2012) y Briceño et al. (2016). En el presente estudio se encontró que los SE más reconocidos por la población se agrupan en 11 categorías; en orden de importancia los más nombrados se ubicaron en la categoría aprovisionamiento, información, hábitat y regulación respectivamente. En la investigación realizada por Briceño et al. (2016) reconocieron 15 funciones ecosistémicas; los SE más nombrados fueron los de información (culturales), seguidos por los de regulación y finalmente por los de aprovisionamiento (abastecimiento).

A escala nacional, la mayoría de los estudios se han destacado más en el enfoque etnobotánico; es decir, hacen un listado de especies en categorías de uso, asimismo describen cómo y porqué diferentes grupos de poblaciones clasifican los elementos del medio ambiente sin agruparlos en servicios ecosistémicos. Algunos ejemplos de estos trabajos son: Martínez et al., 2002; Martínez, Evangelista, Basurto, Mendoza y Cruz, 2007; Jorand, 2008; Martínez, Antonio, Gil y Cuevas, 2012; Gómez et al., 2016; Bautista et al., 2016; Ríos et al., 2017; Zepeda, Burrola, White y Rodríguez, 2017, entre otros. En el caso particular de la descripción de especies y la relación con los nombres científicos, comunes y denominaciones indígenas se encontró el estudio elaborado por Martínez et al. (2002); no obstante, también es un estudio del tipo etnobotánico, por lo que se hizo difícil la comparación con la metodología del presente trabajo.

El artículo presentado contribuye a documentar apropiadamente los SE de la flora existente en las comunidades indígenas, a fin de obtener información básica que ayude a los tomadores de decisiones a facilitar la preparación de estrategias de conservación y de esta forma establecer mecanismos apropiados para su aprovechamiento racional y sustentable (Prieto, 2004; Martínez et al., 2012; Huasasquiche y Kómetter, 2017).

Una limitante en este trabajo es que se concentró en un estrato de edad de la población. Para una futura investigación deben incluirse más estratos, además de usuarios indirectos de los servicios ecosistémicos con el fin de comparar la efectividad de la transmisión de los conocimientos y las diversas valoraciones con diferentes actores involucrados. Del mismo modo, una vez que se ha determinado el valor sociocultural de los ecosistemas, es necesario evaluar los servicios ecosistémicos en términos monetarios a fin de revelar cómo varía la disposición a pagar de los actores involucrados por beneficios que se obtienen del ecosistema.

\section{CONCLUSIONES}

Las comunidades indígenas cuentan con una gran riqueza cultural y de biodiversidad, esto debe ser motivo de orgullo para las nuevas generaciones. La riqueza de conocimientos y la asociación indisoluble entre la cultura y los ecosistemas hace necesario que los estudios sean multidisciplinarios para un mejor acercamiento, conocimiento y comprensión de la forma de aprehender el mundo por parte de dichas comunidades.

En el actual contexto de globalización hace falta el replanteamiento del valor de los servicios ecosistémicos no solo en términos ecológicos o económicos, sino también el aspecto sociocultural para poder avanzar hacia un verdadero desarrollo sustentable con la participación de los actores involucrados; en este sentido, las acciones locales para proteger y restaurar ecosistemas benefician tanto a los usuarios directos como a los indirectos a escala nacional e internacional. 


\section{REFERENCIAS}

Anderson, D., Sweeney, D., \& Williams, T. (2008). Estadística para administración y economía (10 $\mathrm{a}$ ed.). Stamford, Estados Unidos: CENGAGE learning.

Bañuelos F., N., \& Salido A., P. (2007). Consideraciones metodológicas para el diseño de propuestas de desarrollo local/ regional sustentable en comunidades indígenas. $\mathrm{R} a$ Ximhai, 3(1), 27-47.

Bautista G., G., Sol S., Á., Velázquez M., A., \& Llanderal O., T. (2016). Composición florística e importancia socioeconómica de los huertos familiares del Ejido La Encrucijada, Cárdenas, Tabasco. Revista Mexicana de Ciencias Agrícolas, 14(Esp.), 2725-2740.

Briceño, J., Iniguez-Gallardo, V., \& Ravera, F. (2016). Factores que influyen en la percepción de servicios de los ecosistemas de los bosques secos del sur del Ecuador. Ecosistemas, 25(2), 46-58. doi: 10.7818/ECOS.2016.25-2.06

Costanza, R., d'Arge, R., de Groot, R., Farber, S., Grasso, M., Hannon, B., Limburg, K., Naeem, S., O’Neill, R., Paruelo, J., Raskin, R., Sutton, P., \& van den Belt, M. (1997). The value of the world's ecosystem services and natural capital. Nature, 387, 253-260.

Costanza, R., de Groot, R., Sutton, P., van der Ploeg, S., Anderson, S., Kubiszewski, I., Farber, S., \& Turner, R. (2014). Changes in the global value of ecosystem services. Global Environmental Change, 26, 152-158. doi: 10.1016/j.gloenvcha.2014.04.002

Costanza, R., de Groot, R., Braat, L., Kubiszewski, I., Fioramonti, L., Sutton, P., Farber, S., \& Grasso, M. (2017). Twenthy years of ecosystenm services: How far have we come and how far do we still need to go?. Ecosystem Services, 28(PA), 116. doi: 10.1016/j.ecoser.2017.09.008

Daily, G., C. (2000). Management objectives for the protection of ecosystem services. Environmental Science and Policy, 3(2000), 333-339. doi: 10.1016/S1462-9011(00)00102-7

de Groot, R., Wilson, M., \& Boumans, R., M. (2002). A typology for the classification, description and valuation of ecosystem functions, goods and services. Ecological Economics, 41(3), 393-408. doi: 10.1016/S0921-8009(02)00089-7

de Groot, R. (2006). Function analysis and valuation as a tool to assess land use conflicts in planning for sustainable, multifunctional landscapes. Landscape and urban Planning, 75(3), 175-186. doi: 10.1016/j.landurbplan.2005.02.016

Gómez-Baggethun, E., \& de Groot, R. (2007). Capital natural y funciones de los ecosistemas: explorando las bases ecológicas de la economía. Ecosistemas, 16(3), 4-14. doi: $10.7818 /$ re.2014.16-3.00
Gómez G., E., Sol S., Á., García L., E., \& Pérez V., A. (2016). Valor de uso de la flora del Ejido Sinaloa $1^{\text {a }}$ sección, Cárdenas, Tabasco, México. Revista Mexicana de Ciencias Agrícolas, 14(esp.), 2683-2694.

Hein, L., van Koppen, K., de Groot, R., \& van Ierland, E. (2006). Spatial scales, stakeholders and the valuation of ecosystem services. Ecological Economics, 57, 209-228. doi: 10.1016/j.ecolecon.2005.04.005

Huasasquiche S., J., \& Kómetter M., R. (2017). El aporte de los saberes comunales andinos en la utilización de los bienes y servicios ecosistémicos. Estudio de la Mancomunidad Saywite Choquequirao Ampay en Apurimac, Perú. Perú: Programa Bosques Andinos HELVETAS Swiss Intercooperation.

Instituto Nacional de Estadística y Geografía [INEGI]. (2009). Prontuario de información geográfica municipal de los Estados Unidos Mexicanos. Filomeno Mata, Veracrur de Ignacio de la Llave. Recuperado de http://www3.inegi.org.mx/contenidos/app/mexicocifras/ datos_geograficos/30/30067.pdf

Jiménez C., P., Hernández J., M., Espinosa S., G., Mendoza C., G., \& Torrijos A., M. (2015). Los saberes en medicina tradicional y su contribución al desarrollo rural: estudio de caso Región Totonaca, Veracruz. Revista Mexicana de Ciencias Agrícolas, 6(8), 1791-1805.

Jorand, B. (2008). Formas de transformación del conocimiento de la medicina tradicional en los pueblos nahuas del municipio de Hueyapan, Sierra Norte de Puebla. Revista Cuicuilco, 15(44), 181-96.

Martín L., B., Iniesta A., I., García Ll., M., Palomo, I., Casado A., I, et al. (2012). Uncovering Ecosystem Service Bundles through Social Preferences. PLoS ONE, 7(6), e38970. doi:10.1371/journal.pone.0038970

Martínez P., A., Antonio L., P., Gil M., A., \& Cuevas S., J. (2012). Plantas silvestres útiles y prioritarias identificadas en la mixteca poblana, México. Acta Botánica Mexicana, 98, 73-98.

Martínez A., M., Evangelista O., V., Basurto P., F., \& Mendoza C., M. (2002). La etnobotánica y los recursos fitogenéticos: el caso de la Sierra Norte de Puebla. Revista de Geografía Agrícola, 2002, 79-88.

Martínez M., A., Evangelista, V., Basurto, F., Mendoza, M., \& Cruz R., A. (2007). Flora útil de los cafetales en la Sierra Norte de Puebla, México. Revista Mexicana de Biodiversidad, 78, $15-40$.

Millennium Ecosystem Assessment [MEA]. (2005). Ecosystems and Human Well-being: Synthesis. Washington, DC: Island Press. 
Prieto A., M. (2004). "Conocimiento indígena tradicional: el verdadero guardián del oro verde”. Boletín de Antropología Universidad de Antioquia, 18(35), 132-164.

Reyes G., V. \& Martí S., N. (2007). Etnoecología: punto de encuentro entre naturaleza y cultura. Revista Ecosistemas, 16(3), 45-54.

Ríos R., Á., Alanís F., G., \& Favela L., S. (2017). Ethnobotany of vegetal resources, their use and management in Bustamante, Nuevo León State. Revista Mexicana de Ciencias Forestales, 8(44), 1-23. doi: 10.29298/rmcf.v8i44.106

Secretaría de Finanzas y Planeación del Estado de Veracruz [Sefiplan]. (2018). Sistema de información municipal, Cuadernillos municipales 2018. Filomeno Mata. Recuperado de: http:/ / ceieg.veracruz.gob.mx/wpcontent/uploads/sites/21/2018/05/Filomeno-Mata.pdf

Vargas S., G. \& Pire, R. (2017). Inga jinicuil schtdl. Árbol multiuso $\left(1^{\mathrm{a}}\right.$ ed.). Villahermosa, Tabasco: Universidad Juarez Autónoma de Tabasco.
Zepeda G., C., Burrola A., C., White O., L., \& Rodríguez S., C. (2017). Especies leñosas útiles de la selva baja caducifolia en la Sierra de Nanchititla, México. Madera y Bosques, 23(3), 101 119. doi: $10.21829 /$ myb.2017.2331426

Manuscrito recibido el 24 de marzo de 2018

Aceptado el 3 de marzo de 2019

Publicado el 13 de diciembre de 2019

Este documento se debe citar como:

López S., M. A. (2019). La valoración de los servicios ecosistémicos desde la cosmovisión indígena totonaca. Madera y Bosques, 25(3), e2531752. doi: 10.21829/myb.2019.2531752

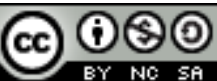

Madera y Bosques por Instituto de Ecología, A.C. se distribuye bajo una Licencia Creative Commons Atribución-NoComercialCompartirlgual 4.0 Internacional.

APÉNDICE 1. Funciones, bienes y servicios de los ecosistemas naturales y seminaturales

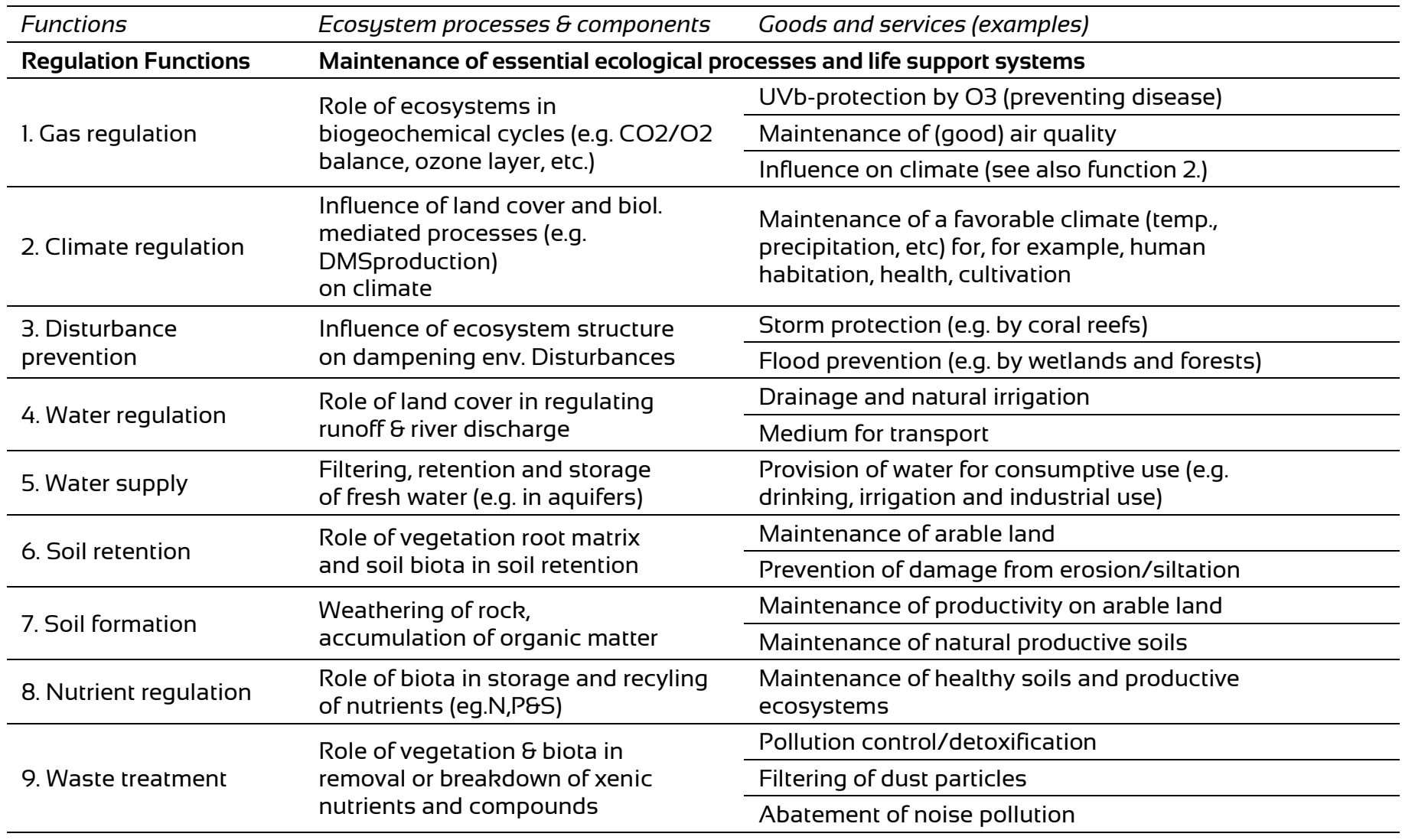




\begin{tabular}{|c|c|c|}
\hline \multirow{2}{*}{ 10. Pollination } & \multirow{2}{*}{$\begin{array}{l}\text { Role of biota in movement of } \\
\text { floral gametes }\end{array}$} & Pollination of wild plant species \\
\hline & & Pollination of crops \\
\hline \multirow{2}{*}{ 11. Biological control } & \multirow{2}{*}{$\begin{array}{l}\text { Population control through } \\
\text { trophic-dynamic relations }\end{array}$} & Control of pests and diseases \\
\hline & & Reduction of herbivory (crop damage) \\
\hline Habitat Functions & \multicolumn{2}{|c|}{ Providing habitat (suitable living space) for wild plant and animal species } \\
\hline 12. Refugium function & $\begin{array}{l}\text { Suitable living space for wild } \\
\text { plants and animals }\end{array}$ & $\begin{array}{l}\text { Maintenance of biological } \& \text { genetic diversity } \\
\text { (and thus the basis for most other functions) }\end{array}$ \\
\hline 13. Nursery Function & Suitable reproduction habitat & Maintenance of commercially harvested species \\
\hline Production Functions & Provision of natural resources & \\
\hline \multirow{2}{*}{ 14. Food } & \multirow{2}{*}{$\begin{array}{l}\text { Conversion of solar energy into } \\
\text { edible plants and animals }\end{array}$} & Hunting, gathering of fish, game, fruits, etc. \\
\hline & & Small-scale subsistence farming $\&$ aquaculture \\
\hline \multirow{3}{*}{ 15. Raw materials } & \multirow{3}{*}{$\begin{array}{l}\text { Conversion of solar energy into } \\
\text { biomass for human construction } \\
\text { and other uses }\end{array}$} & Building \& Manufacturing (e.g. lumber, skins) \\
\hline & & Fuel and energy (e.g. fuel wood, organic matter) \\
\hline & & Fodder and fertilizer (e.g. krill, leaves, litter). \\
\hline \multirow{2}{*}{ 16. Genetic resources } & \multirow{2}{*}{$\begin{array}{l}\text { Genetic material and evolution } \\
\text { in wild plants and animals }\end{array}$} & Improve crop resistance to pathogens \& pests, \\
\hline & & Other applications (e.g. health care) \\
\hline \multirow{3}{*}{ 17. Medicinal resources } & \multirow{3}{*}{$\begin{array}{l}\text { Variety in (bio)chemical substances } \\
\text { in, and other medicinal } \\
\text { uses of, natural biota }\end{array}$} & Drugs and pharmaceuticals \\
\hline & & Chemical models \& tools \\
\hline & & Test- and essay organisms \\
\hline $\begin{array}{l}\text { 18. Ornamental } \\
\text { resources }\end{array}$ & $\begin{array}{l}\text { Variety of biota in natural } \\
\text { ecosystems with (potential) } \\
\text { ornamental use }\end{array}$ & $\begin{array}{l}\text { Resources for fashion, handicraft, jewelry, pets, } \\
\text { worship, decoration } \& \text { souvenirs (e.g. furs, feathers, } \\
\text { ivory, orchids, butterflies, aquarium fish, shells, etc.) }\end{array}$ \\
\hline Information Functions & \multicolumn{2}{|c|}{ Providing opportunities for cognitive development } \\
\hline 19. Aesthetic information & Attractive landscape features & Enjoyment of scenery (scenic roads, housing, etc.) \\
\hline 20. Recreation & $\begin{array}{l}\text { Variety in landscapes with } \\
\text { (potential) recreational uses }\end{array}$ & $\begin{array}{l}\text { Travel to natural ecosystems for eco-tourism, outdoor } \\
\text { sports, etc. }\end{array}$ \\
\hline $\begin{array}{l}\text { 21. Cultural \& artistic } \\
\text { information }\end{array}$ & $\begin{array}{l}\text { Variety in natural features with } \\
\text { cultural and artistic value }\end{array}$ & $\begin{array}{l}\text { Use of nature as motive in books, film, painting, } \\
\text { folklore, national symbols, architect., advertising, etc }\end{array}$ \\
\hline $\begin{array}{l}\text { 22. Spiritual and historic } \\
\text { information }\end{array}$ & $\begin{array}{l}\text { Variety in natural features with } \\
\text { spiritual and historic value }\end{array}$ & $\begin{array}{l}\text { Use of nature for religious or historic purposes (i.e. } \\
\text { heritage value of natural ecosystems and features) }\end{array}$ \\
\hline 23. Science \& Education & $\begin{array}{l}\text { Variety in nature with scientific } \\
\text { and educational value }\end{array}$ & $\begin{array}{l}\text { Use of natural systems for school excursions, etc. } \\
\text { Use of nature for scientific research }\end{array}$ \\
\hline
\end{tabular}

Fuente: de Groot, Wilson y Boumans (2002) 